\title{
Research on Remote Interactive Self-seeking Intelligent Flowerpot Based on Android Client
}

\author{
Xiang $\mathrm{Li}^{\text {a) }}$, Zheng Cui, Hannuo Wang, Fanwei Ma, Yudong Ma \\ Northeast Agricultural University 150036, China. \\ a) Corresponding Author Email: 18846828939@163.com
}

\begin{abstract}
With the continuous development of China's economy and the rise of the Internet of Things technology, today's rising living standards, flower cultivation is increasingly favored by people. However, due to the acceleration of the pace of life, people are often dying because of their busy life and frequent business trips. They are unable to timely water or water the potted plants in their homes or offices or are unreasonable in terms of watering methods and remote access. To solve these problems, based on STM32, a smart pot device for the healthy growth of indoor potted flowers was designed [1]. The smart pots process the collected environmental information through the STM32 module, which mainly includes the collection of soil temperature, humidity, water level in the storage tank, and ambient light intensity. The real-time display of measured data through the LCD screen facilitates human-computer interaction. Based on the detected humidity and light information, the system feeds back feedback through a certain algorithm to automatically control watering and fill light functions on demand, maintain the humidity of the flower soil in the vicinity of the optimal value, and balance the flowers with light to avoid malnutrition. The upper computer adopts C\# language, and the information received by the MCU is saved in the server through the upper computer, and the Android-side login, binding the unique code of the flowerpot [2], obtaining corresponding data, displaying the growth environment on the UI interface, and pushing the growth log daily. Pots work by default when there is no internet connection. The preset value can be adjusted on the Android side to meet the growth habits of different plants. The intelligent flowerpot has a complete set of design functions, which has great aesthetic and practical value for improving the indoor environment, beautifying the urban environment, improving the interest of life, and improving the humanistic qualities of the region. At the same time, it has certain reference and guiding significance for research on precision agriculture in China. The study of wisdom pots can meet people's demand for flowering. It can be seen that the research of pots with wisdom has great development prospects [3].
\end{abstract}

Key words: Sensor, STM32, Android, Information Acquisition, Automatic Control, Remote Access.

\section{RESEARCH PURPOSE}

With the rapid development of our country economy and the improvement of people's awareness of environmental protection, China's flower industry economy is also growing. Family pots have become the new darling of homes and offices today and are increasingly favored by people. However, the fast-paced life has made it difficult to grow flowers easily. Studies have shown that $90 \%$ of the deaths in family pots are caused by improper watering or insufficient lighting. At present, the greening and watering work still depends on manual operations [4]. Because people are busy with work, they often cannot water the pots in time. In addition, due to the limitations of various environmental factors, the uneven illumination of flowers leads to poor nutrition and even death of potted plants. The popularity of smart phones has ushered in the idea of developing a smart flowerpot based on the Android client to realize the networking and intelligence of the flower-planting device [5].

\section{OVERALL DESIGN}

\section{Overall System Process}

The system is divided into two major hardware and software modules, including Android mobile phone, host computer, sensor module, STM32 micro controller, relay module, motor module and buzzer module. The hardware part can be divided into three levels: data acquisition layer, automatic control layer and data transmission layer 
according to the modular design. As shown in Figure 2-1, the sensor data of each environment in the collection site and the real-time display function of the LCD screen can be realized separately. The collected humidity and light information are fed back to realize the function of automatic watering and searching for light, and the function of remotely accessing the collected data through the upper computer and server through the wireless WIFI module.

Each smart pot is equipped with a unique 13-digit code, and the Android-side download is realized by scanning the QR code at the bottom of the flower pot [6]. The Android terminal registers the unique code on the smart pot by scanning the code to obtain the corresponding data. In use, the flower type and preset value can be selected on the Android terminal, transmitted to the server, the server sends to the host computer, and the host computer communicates with the single chip microcomputer to achieve the setting of the optimum environmental value for different types of flower growth on the single chip microcomputer so as to ensure If you do not have a network connection, you can also work by default. The unique code is used as the search code, and the environmental feature values of the flowers are selected in the server at regular intervals. Through the analysis of data processing, the flower environment is divided into four types: normal, abnormal lighting, abnormal temperature, and abnormal humidity. The flower growth $\log$ is generated., Daily push once on the Android side (users can set the push time), to help users complete the adjustment of the flower growth environment. At the same time, when the system is in an abnormal state, if the water level of the water storage tank is close to the lower limit value, the Android terminal will alarm to remind the user to perform the corresponding adjustment operation.
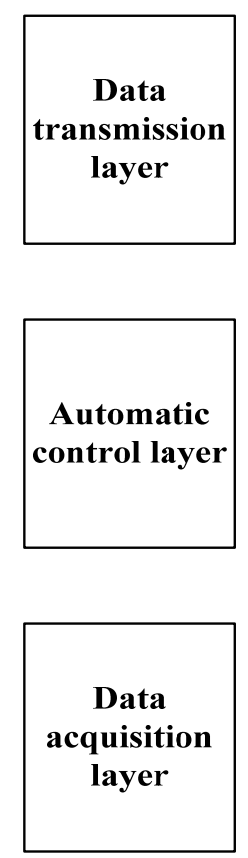
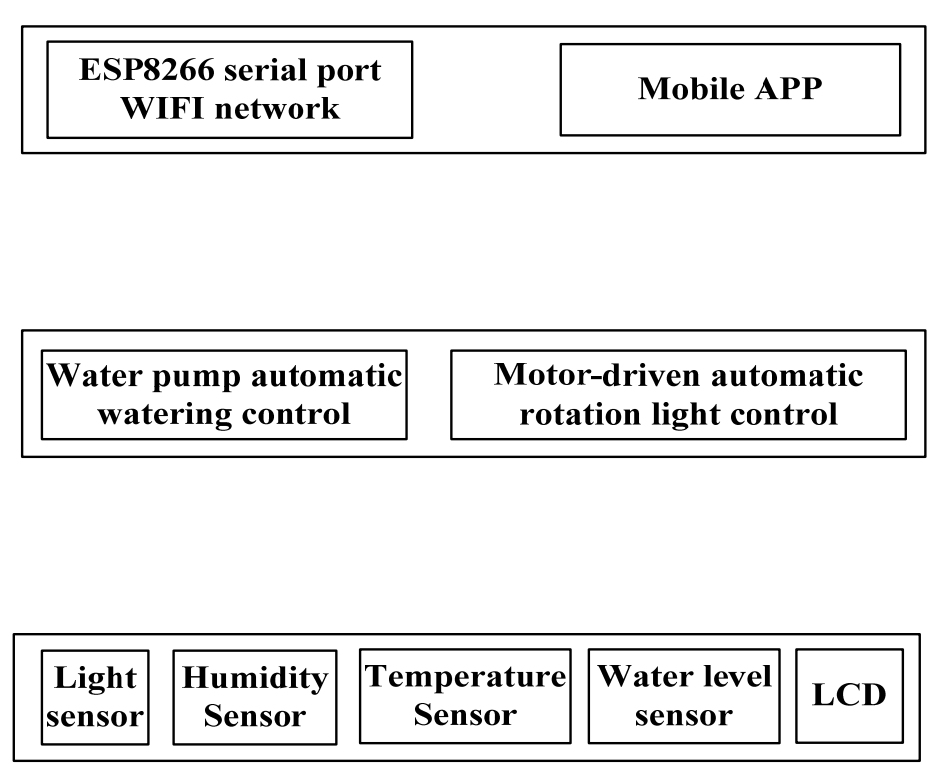

FIGURE 1. Overall hardware architecture. 


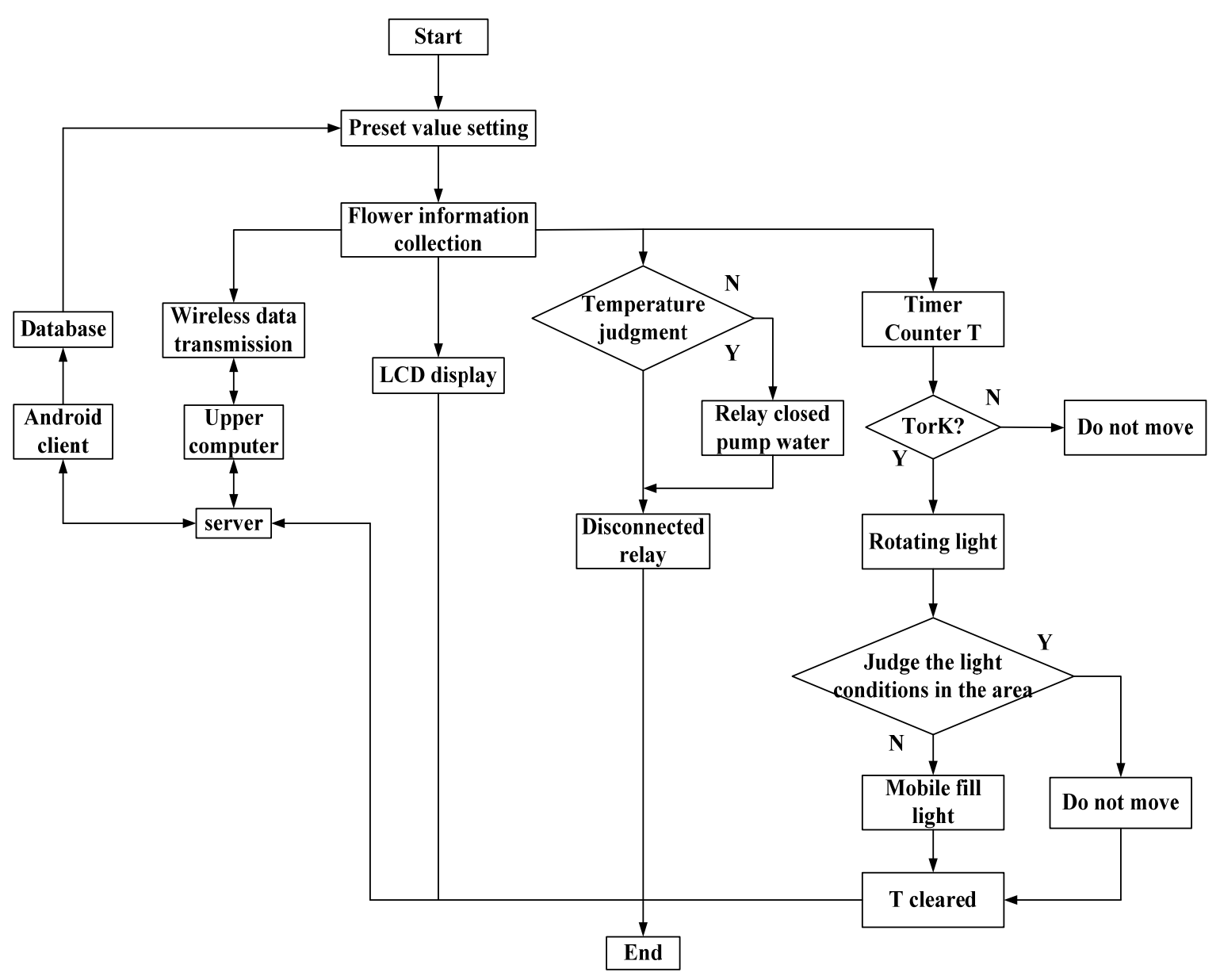

FIGURE 2. Overall Flowchart of Smart Flower Pot.

\section{Software Design}

Functional Definition of UI

The Android side is a collection of interactions between the user and the system. The basic functions of the client are as follows: user registration and login, two-dimensional code scanning, selection of flower types, monitoring of flower growth environment, and log pushing.

Utilize the UI (User Interface) to define the control to complete the three elements of drawing, data and control.

The five functions of the Android interface:

(1) User's registration and login: Download the Android terminal by scanning the QR code on the bottom of the smart flowerpot. After the user starts the user, he first enters the startup interface, and then jumps to the login interface. If you choose to register, the user will jump to the user registration interface. When registering, the user's flower type preference can be selected to push the user with a suitable flowering experience article.

(2) QR code scanning: When a user uses a smart flowerpot for the first time, a QR code can be scanned on the Android device to obtain a unique code as a server search code. The user can also use different smart pots at the same time. Named to achieve different control of multiple flowerpots at the same time.

(3) Selection of flower types: Use UI button controls to realize the selection of different flower types and preset values. Send to the upper computer through the server, and the upper computer communicates with the single-chip microcomputer to realize the same smart flowerpot for multiple flowers. Planting. Moreover, through the programming of the preset value, it can be ensured that the smart pots without network connection can still work 
normally. At the same time, the introduction of selected flowers and planting instructions will be provided in this interface to help users have a better planting experience.

(4) Monitoring the growth environment of flowers: Use UI label controls to display the information of the flower environment obtained through the unique code in the server, such as the parameters of lighting, humidity, temperature, and so on. At the same time, when the smart flower device is in an abnormal state, such as the warning of the water level of the storage tank and the abnormal rotation of the wheel at the bottom of the device, it will also be displayed on this interface to remind the user to take corresponding measures.

(5) Log push: Obtain light abnormality from the server at regular time intervals, take the current flower environment information, and divide the growth environment into normal through the calculation and analysis of the environmental data and the statistics of daily supplemented light. It is divided into four types of abnormal temperature and abnormal humidity. At the same time, a plant growth log is generated. In the evening, it is used to push users to adjust the environment for flower growth. Push the flower planting history report in the form of a table line chart every week or month through the user's settings; at the same time, according to the user's preferences when registering, push the corresponding flowering experience articles from time to time (this interface push function can be customized by the user).

\section{Implementation of MySQL Database}

MySQL is an efficient relational database management system (RDBMS). Relational database is the most common database type at present, and it has a rich theoretical basis. The relational database engine provides more secure and reliable storage functions for data and structural data. MySQL is a mufti-threaded, multi-user server, so the MySQL server can ensure that multiple users have concurrent access to data. Use MySQL to build a relational database to store, manage, and retrieve data that needs to be recorded, such as user registration information, flower growth environment monitoring information, and log information, and operate the database through the Androidside access interface. Real-time data storage, debugging and retrieval at any time.

\section{Hardware Design}

\section{Design and Principle Analysis of Information Acquisition Layer}

The collection layer of the smart flowerpot consists of the STM32 core control chip, information acquisition module, and the LCD display. The information collection part mainly includes a temperature detection module, a humidity detection module, a light detection module, and a water level detection module. The display part is mainly an LCD 9235 display module.

\section{Design and Principle Analysis of Automatic Control Layer}

(1) Design and Principle Analysis of Automatic Control Water Supply Function

In order to maintain the optimum soil moisture for flower growth, the control system uses relays and pumps to achieve automatic water control. The role of the relay module is a switch. Mainly based on the detected humidity information feedback through STM32 to control the valve on and off, and then control the pump water.

(2) Design and Principle Analysis of Automatically Controlling the Quest and Fill Functions

The smart flowerpot feedback-controls the detected light intensity information to drive the rotation of the DC motor. Its algorithm implementation is to divide the circular area of the light sensor detection light into four small areas and collect several numbers for each area during detection. Illumination value, average value is taken as the average light level in this area, it will rotate the weakest light area below the set value to the strongest light area, so that its light is even, to avoid the death of flowers caused by its uneven nutrition. To achieve automatic rotation, the control section uses an L293D DC motor drive module to drive four motor motors for automatic rotation.

\section{Design and Implementation of Data Transmission Layer}

The data transmission layer uses the ESP8266 wireless WIFI module as a medium to connect the MCU and the host computer to realize the storage of information on the server side and facilitate the access of the Android terminal. 


\section{CONCLUSION}

ICT technology has developed rapidly, and home networking is a development trend. The smart flowerpot can achieve remote control of the user through Android. Its main goal is to satisfy the user's analysis and understanding of the environment in which the flowers are growing, to realize that the user can grasp the growth status of the flowers thousands of miles away; to complete the function of automatic watering and automatic fill light, to help the normal growth of flowers, and to improve the indoor environment. The task of beautifying the urban environment. At the same time, this work has certain reference significance for the research of precision agriculture in our country and helps to realize the networking of agricultural facilities.

\section{ACKNOWLEDGEMENTS}

Fund Project: Enterprise-University cooperative Education programmer (201701003077).

\section{REFERENCES}

1. Deng Yangchuan, Xu Songhua, ET al. Review of Soil Moisture Measurement Methods Hydrology, Vol. 14(2007) No. 5, p.21-23.

2. Wei Jingling, ET al. Research and Design of Portable Soil Humidity Detector. Hebei Agricultural University, Vol. 21(2010) No. 9, p.261-265.

3. Li Tianjin, Sun Yuri, Lin Jeanie, ET altar solar powered wireless sensor for soil moisture. Journal of Jiangsu University, Vol. 13(2009) No. 12, p.313-316.

4. Wang Xiao lei, Hu Jading, Jiang Min, et al. Experiment for Rapid Determination of Soil Moisture by Additional Resistance Method. Journal of Agricultural Engineering, Vol. 9 (2007) No.6, p.167-171.

5. Zhang Uele, Hu Zhen qi, Chu Shill, ET al. Advances in soil moisture determination methods. Soil Bulletin, Vol. 7 (2005) No.7, p.332-335.

6. Hu Jading, Zhao Xinyang, Li Zhen Feng, ET al. Parameter Modulation Probe Type Capacitive Soil Moisture Sensing Technology. Journal of Sensing Technology, Vol. 12 (2009) No.3, p.71-75. 